\title{
GMR
}

\section{Development and characterization of microsatellite markers for Solen grandis using Illumina sequencing approach}

\author{
Y.W. Feng ${ }^{1}$, H.L. Jiang ${ }^{1,2}$, Y.T. Liu ${ }^{3}$, X.P. Wu ${ }^{1,4}$, X.Q. Liu ${ }^{1}$ and X.M. Wei ${ }^{1}$ \\ ${ }^{1}$ Key Laboratory of Marine Ecological Restoration, \\ Shandong Marine Resource and Environment Research Institute, Yantai, China \\ ${ }^{2}$ College of Fisheries and Life Science, Shanghai Ocean University, \\ Shanghai, China \\ ${ }^{3}$ College of Agriculture, Ludong University, Yantai, China \\ ${ }^{4}$ College of Life Science and Technology, Guangxi University, Nanning, China \\ Corresponding authors: Y.W. Feng / X.Q. Liu \\ E-mail: fywzxm1228@163.com / 1xq6808@163.com
}

Genet. Mol. Res. 16 (1): gmr16019621

Received January 18, 2017

Accepted January 18, 2017

Published February 8, 2017

DOI http://dx.doi.org/10.4238/gmr16019621

Copyright (C) 2017 The Authors. This is an open-access article distributed under the terms of the Creative Commons Attribution ShareAlike (CC BY-SA) 4.0 License.

\begin{abstract}
Solen grandis is an important economic and overexploited bivalve species. In order to perform its fine-scale genetic analyses, 105 pairs of microsatellites with polymorphism were identified through Illumina Hiseq platform and bioinformatic assembly technology in this study. The estimated fragment size ranged from 100 to $268 \mathrm{bp}$ and the number of alleles per locus varied between 2 and 23. Observed and expected heterozygosities varied from 0.0667 to 1.0000 and 0.0966 to 0.9492 , respectively. Fourteen loci deviated significantly from HardyWeinberg equilibrium after Bonferroni correction. These microsatellite markers developed in this study would be helpful for future genetic studies on $S$. grandis and closely related species.
\end{abstract}

Key words: Solen grandis; Microsatellite; Illumina sequencing

Genetics and Molecular Research 16 (1): gmr16019621 


\section{INTRODUCTION}

Solen grandis (Dunker, 1861), a bivalve mollusk of the family Solenidae, has become an important cultured species in China due to its high nutritive (Dai, 2002), high medical (Yang and Wang, 1996) and high economic value (Yuan et al., 2010). However, population decline caused by overfishing and deterioration of marine environment have limited the aquaculture industry. Under the consideration of restoration and promising strain selection, related genetic analyses should be performed. Microsatellite loci, also known as simple sequence repeats (SSR), are widely used for detecting genetic diversity, association mapping, genetic linkage mapping, and population and evolution analysis owing to their advantages such as hypervariability, reproducibility, co-dominant inheritance, and extensive genomic coverage (Powell et al., 1996; Jiao et al., 2012). So far, some microsatellite markers have been isolated for $S$. grandis (Yuan et al., 2010; Jiang et al., 2016), but these do not satisfy the need of genetic studies and the development of its industry.

The purpose of this study was to use the next-generation sequencing (NGS) method (Illumina), which is more cost-effective and higher in throughput, to develop novel microsatellite markers for $S$. grandis. These new loci will greatly facilitate future genetic studies and brood stock management.

\section{MATERIAL AND METHODS}

Thirty wild $S$. grandis individuals were sampled successfully from Yantai in Shandong, China. Adductor muscles were collected and stored in ethanol, under $-20^{\circ} \mathrm{C}$ condition. The modified phenol-chloroform protocol ( $\mathrm{Li}$ et al., 2006) was used to extract genomic DNA. Genomic DNA quality and quantity were detected with the NanoDrop 2000 (Thermo Scientific, Wilmington, DE, USA) and $1.0 \%$ agarose gel.

Extracted DNA of one individual was randomly shotgun for specific fragments. The terminal of the fragments was repaired and 3' end was ligated with the A base. After combining with sequencing primers, approximately $170-\mathrm{bp}$ fragments were purified, and then the library was constructed. The sequencing was performed on Illumina Hiseq 2000 platform.

The obtained sequence data were filtered and then subject to assembly. Microsatellite loci were identified using SSRHunter ( $\mathrm{Li}$ and Wan, 2005). The criteria used in SSRHunter were as follows: five repeats for di-, tri-, tetra- and pentanucleotide repeats. Those loci with enough flanking sequence were used for primer design using PRIMER 5.0 (http://www. premierbiosoft.com/). Each pair of primers was tested on $30 \mathrm{~S}$. grandis individuals. PCR amplifications were performed in a reaction mixture $(10 \mu \mathrm{L})$ containing 50 ng genomic DNA, 1X PCR Buffer ( $\mathrm{Mg}^{2+}$ plus), $0.2 \mathrm{mM}$ of each dNTP, $0.25 \mathrm{U}$ DNA ploymerase (TaKaRa) and $1 \mu \mathrm{M}$ primer set. The thermo-cycling conditions were as follows: 3 min at $94^{\circ} \mathrm{C}, 35$ cycles of $45 \mathrm{~s}$ at $94^{\circ} \mathrm{C}, 45 \mathrm{~s}$ at primer specific annealing, $45 \mathrm{~s}$ at $72^{\circ} \mathrm{C}$ and a final elongation of $5 \mathrm{~min}$ at $72^{\circ} \mathrm{C}$. PCR products were genotyped by polyacrylamide gel and allele size was determined by a reference standard 10-bp ladder marker (Invitrogen).

Polymorphism information content (PIC) was calculated by program CERVUS 3.0 (Marshall et al., 1998). Number of alleles per locus $\left(N_{\mathrm{A}}\right)$, observed heterozygosities $\left(H_{\mathrm{O}}\right)$ and expected heterozygosities $\left(H_{\mathrm{E}}\right)$ were analyzed by the Microsatellite Analyzer software (Dieringer and Schlötterer, 2003). Deviations from Hardy-Weinberg equilibrium (HWE) and tests for linkage disequilibrium were performed by GENEPOP (Rousset, 2008).

Genetics and Molecular Research 16 (1): gmr16019621 


\section{RESULTS AND DISCUSSION}

It is well known that the development of microsatellite markers using enrichment techniques requires a great amount of effort. The NGS provides a more cost-effective and higher throughput method to generate this useful marker (Ji et al., 2012). In this study, we used Illumina HiSeq 2000 platform to obtain $27,253.71 \mathrm{Mb}$ clean data, which were assembled into 46,715 contigs $(\geq 1 \mathrm{~kb})$ and 46,877 scaffolds $(\geq 1 \mathrm{~kb})$, respectively.

From 250 loci we selected for microsatellite marker optimization, we found a total of 105 loci that were successfully amplified and proven to be polymorphic on 30 samples. It includes 50 dinucleotides, 43 trinucleotides, 10 tetranucleotides and 2 pentanucleotides (Table 1). The mean number of individuals successfully genotyped was 29.5 , which indicates excellent amplification efficiency of these primers. $N_{\mathrm{A}}$ ranged from 2 to 23 , with mean number of 8 , and allele size varied between 100 and $268 \mathrm{bp} . H_{\mathrm{O}}$ and $H_{\mathrm{E}}$ ranged from 0.0667 to 1.0000 and 0.0966 to 0.9492 , respectively. Fourteen loci significantly deviated from HWE after sequential Bonferroni correction $(\mathrm{P}<0.05 / 105)$, probably because of null alleles or excessive heterozygosity. No linkage disequilibrium between each of loci was found. In addition, PIC values ranged from 0.090 to 0.929 . According to Botstein et al. (1980), 103 loci of our study were highly or reasonably informative (PIC > 0.25).

\begin{tabular}{|c|c|c|c|c|c|c|c|c|c|c|}
\hline Locus & Primer sequence $\left(5^{\prime}-3^{\prime}\right)$ & Repeat motif & Fragment size (bp) & $\mathrm{Ta}\left({ }^{\circ} \mathrm{C}\right)$ & $N_{\mathrm{A}}$ & $H_{0}$ & $H_{\mathrm{E}}$ & PIC & P value & GenBank accession No. \\
\hline$\overline{\mathrm{DZC} 341}$ & $\begin{array}{l}\text { F:ACCTGCCGATAATGAGTGTTTCAT } \\
\text { R:AAAGGGAGTAAGTCCTGGGTTCAC }\end{array}$ & $\mathrm{CA}_{(8)}$ & $124-158$ & 59 & 5 & 0.6333 & 0.6944 & 0.764 & 0.0373 & KX530218 \\
\hline$\overline{\text { DZC334 }}$ & $\begin{array}{l}\text { F:CTTTAAAGGTCACACCCGATGTCT } \\
\text { R:GCTGAAGTAGGTGTTTGATTTCATTG }\end{array}$ & $\mathrm{CAT}_{(8)}$ & 134-198 & 59 & 12 & 0.6333 & 0.8333 & 0.887 & 0.0588 & KX530219 \\
\hline$\overline{\mathrm{DZC} 242}$ & \begin{tabular}{|l|} 
F:CCCTGTAAATGCCAATGAATATTTTA \\
R:CCCTGTTTATATTTTGAATTGTGTATGA
\end{tabular} & ATTC $(4)_{1}$ & $160-172$ & 59 & 10 & 0.6667 & 0.8650 & 0.614 & 0.0060 & KX530220 \\
\hline$\overline{\text { DZC236 }}$ & \begin{tabular}{|l|} 
F:GGGTTTAGTTTAACGATTGAAAAGGG \\
R:TTGAAAACAGTTATGGTGGTGATGA
\end{tabular} & $\mathrm{TCC}_{(7)}$ & $130-138$ & 59 & 10 & 0.5667 & 0.8073 & 0.682 & 0.1903 & KX530221 \\
\hline DZC239 & $\begin{array}{l}\text { F:AGTAAATTTCCCTATTCGTGGGCT } \\
\text { R:GAGCACAATACACAACAGGTCGTT }\end{array}$ & $\mathrm{AC}_{(13)}$ & $116-138$ & 59 & 21 & 0.8333 & 0.9367 & 0.826 & 0.0173 & KX530222 \\
\hline DZC156 & $\begin{array}{l}\text { F:GTGATGGCACTCTGTTTTTCTACTTAAA } \\
\text { R:TAGGGCATTCTCAAAGAGATGGAG }\end{array}$ & $\mathrm{TC}_{(18)}$ & $122-154$ & 59 & 8 & 0.9000 & 0.8006 & 0.850 & 0.0592 & KX530223 \\
\hline$\overline{\text { DZC143 }}$ & $\begin{array}{l}\text { F:TGACTAAAACTCACGATCGAAAGAAC } \\
\text { R:AGCATGCCACCGAATATCTTTTTA }\end{array}$ & $\mathrm{CA}_{(22)}$ & $134-172$ & 59 & 13 & 0.7000 & 0.8977 & 0.899 & 0.0128 & KX530224 \\
\hline DZC159 & $\begin{array}{l}\text { F:AGAAAACAACAACAGACGGACGAT } \\
\text { R:CTGCAGTGTGGCAGCACTATATCT }\end{array}$ & $\operatorname{ATAG}_{(12)}$ & $100-126$ & 59 & 13 & 0.7667 & 0.8921 & 0.786 & $0.0002^{*}$ & KX530225 \\
\hline DZC153 & $\begin{array}{l}\text { F:GGTATGCATCATACATCGGATACCT } \\
\text { R:GCACCAGAACAACAAACAAATGAG }\end{array}$ & $\mathrm{TG}_{(18)}$ & $130-176$ & 59 & 13 & 0.8000 & 0.9068 & 0.928 & 0.0668 & KX530226 \\
\hline DZC400 & $\begin{array}{l}\text { F:GAATACACATTCGTGGGTTGTTGGA } \\
\text { R:TAGAGGACAAGAGTGGTCTCCACC }\end{array}$ & $\mathrm{AG}_{(6)}$ & $180-224$ & 59 & 14 & 0.9667 & 0.9249 & 0.903 & 0.8156 & KX530227 \\
\hline$\overline{\mathrm{DZC} 116}$ & $\begin{array}{l}\text { F:TTTTTCGAATGCTATCACTGTTTCC } \\
\text { R:AAGCCTATGCACAGTTCTTTTGC }\end{array}$ & $\mathrm{TA}_{(10)}$ & $112-134$ & 57 & 10 & 0.9000 & 0.8599 & 0.769 & 0.9892 & KX530228 \\
\hline DZC177 & $\begin{array}{l}\text { F:CACATAAAGCTTCCAAAATAGGGGG } \\
\text { R:GCAAAACGGTAAACGCTTTCATAC }\end{array}$ & $\mathrm{AC}_{(8)}$ & $146-184$ & 57 & 11 & 0.7667 & 0.8887 & 0.929 & 1.0000 & KX530229 \\
\hline DZC163 & $\begin{array}{l}\text { F:CGTATCTTGTCTTGTGGAATACGTG } \\
\text { R:AAAATGTTGCTAACCCATTTTTGC }\end{array}$ & $\mathrm{AC}_{(19)}$ & $120-152$ & 55 & 7 & 0.5000 & 0.7605 & 0.909 & 0.3534 & KX530230 \\
\hline DZC234 & $\begin{array}{l}\text { F:CACGAAAAGACGATGTCGAAACTA } \\
\text { R:TTTAAGGGGTGTGTCAAATTACGC }\end{array}$ & $\mathrm{AG}_{(20)}$ & $128-150$ & 60 & 13 & 0.8000 & 0.8989 & 0.817 & 0.1543 & KX530231 \\
\hline$\overline{\text { DZC } 350}$ & $\begin{array}{l}\text { F:TGGTCTAAATGCATTGTTTTCTGTG } \\
\text { R:CCGCACCAACTAGTCTTCTGACTT }\end{array}$ & $\operatorname{AAAT}_{(5)}$ & $112-130$ & 59 & 18 & 1.0000 & 0.9316 & 0.794 & 0.6834 & KX530232 \\
\hline$\overline{\text { DZC240 }}$ & $\begin{array}{l}\text { F:GGGTACGGATCGTAACTGATCTCTC } \\
\text { R:AATAAACGAAAAACCGAGTGCAAA }\end{array}$ & $\mathrm{AC}_{(16)}$ & $110-138$ & 63 & 8 & 0.6000 & 0.7588 & 0.879 & $0.0000^{*}$ & KX530233 \\
\hline DZC395 & $\begin{array}{l}\text { F:CGAGTTATATGCATAATTTAGCTGTGC } \\
\text { R:AATGCTCTCAACATTCAACTCACAA }\end{array}$ & $\mathrm{AC}_{(7)}$ & $142-168$ & 63 & 13 & 0.2667 & 0.9056 & 0.875 & 0.0153 & KX530234 \\
\hline DZC283 & $\begin{array}{l}\text { F:CCTTCGTTTTCAAACACTTAGGAGA } \\
\text { R:GAGTACACCGGATGATTAAATGGG }\end{array}$ & $\mathrm{TG}_{(13)}$ & $106-128$ & 61 & 12 & 0.7333 & 0.8644 & 0.882 & 0.1010 & KX530235 \\
\hline$\overline{\text { DZC381 }}$ & \begin{tabular}{|l} 
F:TTCGCCACTTTTCAATGACATCTA \\
R:TTGGGACAAATGCACTCTATCATC
\end{tabular} & $\mathrm{AG}_{(13)}$ & $114-140$ & 63 & 9 & 0.7333 & 0.8407 & 0.866 & 0.0008 & KX530236 \\
\hline DZC288 & $\begin{array}{l}\text { F:TAAAGCGCGTTCTAATGGTTCTTC } \\
\text { R:AACAACACATTGCAACAATAACTGC }\end{array}$ & $\mathrm{AGT}_{(10)}$ & $150-168$ & 57 & 13 & 0.6000 & 0.9068 & 0.873 & $0.0000^{*}$ & KX530237 \\
\hline$\overline{\mathrm{DZC} 292}$ & $\begin{array}{l}\text { F:AAGTACATGCGTGGATTCAGAAGG } \\
\text { ::GACAACGACGAATCGAATACCTG }\end{array}$ & $\mathrm{CA}_{(18)}$ & $140-170$ & 64 & 12 & 0.7000 & 0.8915 & 0.903 & 0.0500 & KX530238 \\
\hline DZC328 & $\begin{array}{l}\text { F:CCGTTTAAAAATAAATCCAACGCA } \\
\text { R:CAGGCAAGTAAAAATAGCTGGATGA }\end{array}$ & $\mathrm{AC}_{(15)}$ & $130-160$ & 55 & 4 & 0.1667 & 0.4164 & 0.902 & 0.6560 & KX530239 \\
\hline DZC216 & $\begin{array}{l}\text { F:CTTCACACAGCACCATCAACATC } \\
\text { R:CTGAGAATGTTGCTGAGGCATACT }\end{array}$ & $\mathrm{ACA}_{(9)}$ & $130-134$ & 60 & 6 & 0.3333 & 0.6017 & 0.792 & 0.0027 & KX530240 \\
\hline
\end{tabular}

Continued on next page

Genetics and Molecular Research 16 (1): gmr16019621 
Y.W. Feng et al.

Table 1. Continued.

\begin{tabular}{|c|c|c|c|c|c|c|c|c|c|c|}
\hline Locus & Primer sequence $\left(5^{\prime}-3^{\prime}\right)$ & Repeat motif & Fragment size (bp) & $\mathrm{Ta}\left({ }^{\circ} \mathrm{C}\right)$ & $N_{\mathrm{A}}$ & $H_{\mathrm{o}}$ & $H_{\mathrm{E}}$ & PIC & P value & GenBank accession No. \\
\hline$\overline{\mathrm{DZC} 330}$ & $\begin{array}{l}\text { F:CCGTTTAAAAATAAATCCAACGCA } \\
\text { R:CAGGCAAGTAAAAATAGCTGGATGA }\end{array}$ & $\operatorname{ACAT}_{(5)}$ & $128-188$ & 55 & 2 & 0.5667 & 0.4130 & 0.868 & 0.7952 & KX530241 \\
\hline$\overline{\mathrm{DZC} 275}$ & $\begin{array}{l}\text { F:GGCATCAATATTTTGCTGAATAGC } \\
\text { R:AGATTTAGGTTACACACATGAGAGAGG }\end{array}$ & $\mathrm{CT}_{(9)}$ & $140-160$ & 55 & 3 & 0.9667 & 0.5407 & 0.845 & 0.0038 & KX530242 \\
\hline DZC245 & \begin{tabular}{|l|} 
F:CCAATTTTCGAATGTTTGGATGAT \\
R:TTAAAATTGGATGCATATCGAGGG
\end{tabular} & $\mathrm{AC}_{(16)}$ & $138-166$ & 55 & 9 & 0.5667 & 0.5853 & 0.882 & $0.0000^{*}$ & KX530243 \\
\hline$\overline{\text { DZC183 }}$ & $\begin{array}{l}\text { F:CATGCATTAAAGTACATATGGAAACAGA } \\
\text { R:TTCGACATTGAGAAAGTGTTGTGA }\end{array}$ & $\mathrm{AC}_{(18)}$ & $122-148$ & 54 & 4 & 0.1333 & 0.5435 & 0.828 & 0.8712 & KX530244 \\
\hline DZC189 & \begin{tabular}{|l} 
F:TTCCTCGCAATATGCCTAAAGATA \\
R:TGGAAGAAGTCTATTATTCATAACATGGG
\end{tabular} & $\mathrm{AG}_{(9)}$ & 148-182 & 54 & 5 & 0.3000 & 0.5073 & 0.861 & 0.0788 & KX530245 \\
\hline$\overline{\mathrm{DZC} 294}$ & \begin{tabular}{|l|} 
F:GTTTGTAGTGCTCCGACCCTGAAT \\
R:TGGCCCCTACTCAAATTTGTTTTT
\end{tabular} & GGTA(4) & $166-186$ & 57 & 5 & 0.6333 & 0.6062 & 0.826 & 0.7881 & KX530246 \\
\hline DZC263 & $\begin{array}{l}\text { F:CCTTGAGCTTGTCTGTCTCTTTCC } \\
\text { R:AAAACTCTAATATGGCACCCCGTT }\end{array}$ & $\operatorname{ATC}_{(\vartheta)}$ & $132-160$ & 56 & 2 & 0.3000 & 0.3045 & 0.736 & $0.0000^{*}$ & KX530247 \\
\hline DZC308 & $\begin{array}{l}\text { F:TCTGGAATGTAGAACCAATATCTCTCA } \\
\text { R:AAAAATAAGAAATGGAGAGTGGGC }\end{array}$ & $\mathrm{TC}_{(20)}$ & $194-228$ & 57 & 8 & 0.7667 & 0.7085 & 0.893 & 0.0737 & KX530248 \\
\hline$\overline{\mathrm{DZC} 314}$ & $\begin{array}{l}\text { F:GTCCAAACCGAAGCAGAGGTATC } \\
\text { R:GCATAAGATGCGAGGAACATTTTT }\end{array}$ & $\operatorname{TTTA}_{(4)}$ & $142-184$ & 57 & 5 & 0.1667 & 0.7768 & 0.929 & 0.0153 & KX530249 \\
\hline$\overline{\mathrm{DZC} 445}$ & $\begin{array}{l}\text { F:TGATGGTACATCAGCGTAGAGCTAAA } \\
\text { R:CAAGGGTTGGCAAAATTCAAGTAA }\end{array}$ & $\mathrm{AG}_{(21)}$ & $140-176$ & 57 & 7 & 0.7000 & 0.7266 & 0.899 & 0.0062 & KX530250 \\
\hline$\overline{\text { DZC } 323}$ & $\begin{array}{l}\text { F:ATTAATTTCTTCTGCTGCGTCCAA } \\
\text { R:TATTGAGAAGGCAGAGAATGGAGC }\end{array}$ & $\mathrm{TCC}_{(6)}$ & $144-156$ & 57 & 6 & 0.6000 & 0.7311 & 0.382 & 0.0101 & KX530251 \\
\hline$\overline{\mathrm{DZC} 279}$ & $\begin{array}{l}\text { F:CATCGTAAATGACACGATTTTGGA } \\
\text { R:GCAATATGGTTGAAATATTGCCGT }\end{array}$ & TTG $_{(5)}$ & $140-150$ & 57 & 5 & 0.3000 & 0.4045 & 0.263 & $0.0004^{*}$ & KX530252 \\
\hline$\overline{\mathrm{DZC} 391}$ & \begin{tabular}{|l|} 
F:TTTCGTATGGTTTCTTGCACTTGA \\
R:GTCTATGGGAACACCGGTTGTAAA
\end{tabular} & $\operatorname{ATC}_{(5)}$ & $140-156$ & 57 & 6 & 0.2667 & 0.3305 & 0.711 & 0.0141 & KX530253 \\
\hline DZC410 & $\begin{array}{l}\text { F:GGTCAAAGCAATGTACTGGCTACC } \\
\text { R:TTTGCGAAATTAACAACTCAAAGACA }\end{array}$ & $\mathrm{GTT}_{(7)}$ & $106-154$ & 56 & 6 & 0.3667 & 0.6198 & 0.742 & 0.0028 & KX530254 \\
\hline$\overline{\mathrm{DZC}} 225$ & \begin{tabular}{|l|} 
F:TAGCTGAATCAAATTCTGCCATGT \\
R:ATTGGTTCCTGGAATTTTGTGAGT
\end{tabular} & $\mathrm{GT}_{(12)}$ & $134-176$ & 52 & 6 & 0.6333 & 0.6463 & 0.856 & 0.0026 & KX530255 \\
\hline DZC417 & $\begin{array}{l}\text { F:GGGGTTTGGCAATTACATTAAGAG } \\
\text { R:TACTTCCCACGTCGTCTGTTATGA }\end{array}$ & $\mathrm{ACC}_{(4)}$ & $153-168$ & 59 & 6 & 0.5000 & 0.5328 & 0.090 & 1.0000 & KX530256 \\
\hline DZC397 & $\begin{array}{l}\text { F:AACGTTTTCCTCACTTGAAGTTGC } \\
\text { R:AGTTTGTCTCGCTTTACAGATCG }\end{array}$ & TG(16) & $104-130$ & 59 & 5 & 0.5333 & 0.6780 & 0.891 & 0.7227 & KX530257 \\
\hline$\overline{\text { DZC398 }}$ & $\begin{array}{l}\text { F:GCTCATATGTTGCATTTTTGGACA } \\
\text { R:CTTGCAGCATAGGTGCATTAAAAAA }\end{array}$ & $\mathrm{TG}_{(7)}$ & $100-122$ & 57 & 5 & 0.2667 & 0.4672 & 0.816 & 0.0022 & KX530258 \\
\hline DZC435 & $\begin{array}{l}\text { F:CGTTACAACAGGGTCGTATCACTG } \\
\text { R:AAAAGCTGATAAGGAAAGGCAAGG }\end{array}$ & $\mathrm{TC}_{(7)}$ & $140-166$ & 59 & 4 & 0.4667 & 0.5249 & 0.809 & 0.0021 & KX530259 \\
\hline DZC439 & $\begin{array}{l}\text { F::TGGTTCACCTTGATAACATTTCCA } \\
\text { R:ACAGGGTCTGTCGAAGATCTGAAC }\end{array}$ & $\begin{array}{ll}\mathrm{CT}(8) \\
\end{array}$ & 106-130 & 57 & 5 & 0.2667 & 0.7350 & 0.873 & 0.2359 & KX530260 \\
\hline$\overline{\text { DZC446 }}$ & $\begin{array}{l}\text { F:AGACAGGTGTACGTTCCTTTCCTG } \\
\text { R:AATGGAAGACAACTTCCGAACCTT }\end{array}$ & $\mathrm{AC}_{(14)}$ & $124-164$ & 59 & 3 & 0.4667 & 0.5249 & 0.910 & 1.0000 & KX530261 \\
\hline DZC337 & $\begin{array}{l}\text { F:AAGGGAATAGTTTGATGCATTTGG } \\
\text { R:AGATTATCCAGTTTCGGTGGATTC }\end{array}$ & $\operatorname{TTG}_{(4)}$ & $160-178$ & 58 & 4 & 0.4667 & 0.5390 & 0.719 & 0.0950 & KX530262 \\
\hline DZC201 & $\begin{array}{l}\text { F:TGCAATGTACAAAGGAAAACAAAAAA } \\
\text { R:TAAAACCCAAACTAGAGCATTGGC }\end{array}$ & $\mathrm{AAC}_{(5)}$ & $118-150$ & 58 & 2 & 0.4000 & 0.5085 & 0.881 & $0.0000^{*}$ & KX530263 \\
\hline DZC126 & $\begin{array}{l}\text { F:AAGAAGGCTTATCTTCGCTCTTGA } \\
\text { R:AGGCAGATAAATGAGCTAGTCGGT }\end{array}$ & $\mathrm{TG}_{(16)}$ & $100-114$ & 58 & 7 & 0.2667 & 0.7921 & 0.645 & 1.0000 & KX530264 \\
\hline DZC372 & $\begin{array}{l}\text { F:ATGCGTTTTCCTCTTCTATCTCGT } \\
\text { R:AAAGTCAAGGTCATCAAAATGACATAC }\end{array}$ & $\mathrm{TC}_{(7)}$ & $136-160$ & 60 & 8 & 0.2333 & 0.2780 & 0.806 & 0.9491 & KX530265 \\
\hline$\overline{\mathrm{DZC}} 421$ & $\begin{array}{l}\text { F:GATTCATGAAAAGCCATCCGATAC } \\
\text { R:TCTTCGAAAAACAGACGAGCTAAAA }\end{array}$ & $\mathrm{CA}_{(16)}$ & $134-148$ & 58 & 5 & 0.2333 & 0.4260 & 0.847 & 0.2115 & KX530266 \\
\hline$\overline{D Z C 169}$ & $\begin{array}{l}\text { F:ACAATGAATGCGTATAATGTAAACGTG } \\
\text { R:AAAAATCGTAAGCTGGACTGTTGG }\end{array}$ & $\mathrm{TC}_{(15)}$ & $110-136$ & 55 & 6 & 0.2667 & 0.4627 & 0.805 & 0.0008 & KX530267 \\
\hline$\overline{\mathrm{DZC} 425}$ & \begin{tabular}{|l|} 
F:TACAGCCTCTCTCATTCTCCCAAT \\
R:AGACTTGACCTCGAAATTCAATGC
\end{tabular} & $\begin{array}{ll}\mathrm{CT}_{(6)} \\
\end{array}$ & $130-146$ & 63 & 5 & 0.0667 & 0.4910 & 0.745 & 0.0018 & KX530268 \\
\hline$\overline{\mathrm{DZC} 345}$ & $\begin{array}{l}\text { F:GCGTTTAAAGGATACACATTTCGC } \\
\text { R:ACGGTTAAATGAGCCATAACTCCA }\end{array}$ & $\operatorname{AAT}_{(10)}$ & $124-148$ & 61 & 6 & 0.3667 & 0.7124 & 0.865 & 0.0031 & KX530269 \\
\hline DZC247 & $\begin{array}{l}\text { F:GCGGGTGAGCAGACTAATGTTAAAA } \\
\text { R:GGCAATACCCTTTTGTCATGTGAT }\end{array}$ & TTGT $_{(5)}$ & $140-146$ & 59 & 11 & 0.6667 & 0.7531 & 0.346 & 0.1438 & KX530270 \\
\hline$\overline{\mathrm{DZC} 112}$ & $\begin{array}{l}\text { F:TAGCCAATGAAAGACTGCGTAATG } \\
\text { R:TGACTGCACAAAATCCAGTGAAAT }\end{array}$ & $\operatorname{TTG}_{(7)}$ & 104-114 & 59 & 17 & 0.7000 & 0.8802 & 0.507 & 0.0453 & KX530271 \\
\hline$\overline{\text { DZC333 }}$ & $\begin{array}{l}\text { F:CGGGTAAAACACTTATATCACTCGC } \\
\text { R:CGTCCTTATTTGCTGTACTTTCTTCA }\end{array}$ & $\operatorname{AGAT}_{(17)}$ & $120-130$ & 59 & 7 & 0.4333 & 0.5955 & 0.378 & 0.0005 & KX530272 \\
\hline DZC246 & $\begin{array}{l}\text { F:TATATGGTCGATATGACGCAAAGC } \\
\text { R:CCCACTCCCACTGAAACTTAACAT }\end{array}$ & $\mathrm{CCAA}_{(6)}$ & $102-118$ & 59 & 17 & 0.7667 & 0.9181 & 0.367 & 0.0495 & KX530273 \\
\hline$\overline{\text { DZC171 }}$ & $\begin{array}{l}\text { F:GAAACACGACGCCATTATAAATCTG } \\
\text { R:TCTGGATCTTGAAATGTAGTAAATCCG }\end{array}$ & $\mathrm{AC}_{(15)}$ & $150-162$ & 59 & 16 & 0.9333 & 0.9198 & 0.535 & 0.0005 & KX530274 \\
\hline$\overline{\text { DZC191 }}$ & $\begin{array}{l}\text { F:GCAAAATATGCAAAATTATCGTGA } \\
\text { R:CAATGTATTACTGAAGCAGCCAAG }\end{array}$ & $\mathrm{TTG}_{(17)}$ & 196-208 & 59 & 19 & 1.0000 & 0.9492 & 0.309 & 0.0318 & KX530275 \\
\hline DZC202 & $\begin{array}{l}\text { F:TCTTCTGATGAAGTAACATTTGCG } \\
\text { R:CATTTTGATCATTTTCGTACACACA }\end{array}$ & $\operatorname{TGTC}_{(5)}$ & $152-168$ & 59 & 16 & 0.9000 & 0.9311 & 0.576 & 0.0134 & KX530276 \\
\hline$\overline{\mathrm{DZC} 162}$ & $\begin{array}{l}\text { F:TTAAATTGAAGTTTAGAAATACGGCACA } \\
\text { R:GTCAGACAAGCTGGATGTCGAGT }\end{array}$ & $\mathrm{AG}_{(10)}$ & $130-136$ & 59 & 13 & 0.6667 & 0.8740 & 0.324 & 1.0000 & KX530277 \\
\hline DZC115 & $\begin{array}{l}\text { F:AGTTCTTGTCACTGCCCAATTTTA } \\
\text { R:TCCACTTTATATTTACGTCATTGTGTTT }\end{array}$ & $\mathrm{TC}_{(11)}$ & $114-122$ & 59 & 11 & 0.7667 & 0.8480 & 0.421 & 0.3994 & KX530278 \\
\hline$\overline{\text { DZC140 }}$ & $\begin{array}{l}\text { F:TGAGTATCTTCAACCAACTCCATTCA } \\
\text { R:GCAGTAAACCACCCAAAAGCATAA }\end{array}$ & $\mathrm{TC}_{(19)}$ & 108-126 & 59 & 9 & 0.8667 & 0.8305 & 0.570 & 0.0791 & KX530279 \\
\hline$\overline{\text { DZC218 }}$ & $\begin{array}{l}\text { F:TCCAGGTGATACTGAAGCTATGGA } \\
\text { R:GGCAGATGGTGCAGTAGAATGTTA }\end{array}$ & $\mathrm{GAT}_{(4)}$ & $156-170$ & 60 & 14 & 0.8000 & 0.8927 & 0.745 & 0.0080 & KX530280 \\
\hline DZC335 & $\begin{array}{l}\text { F:CTGCAGTGTGGCAGCACTATATCT } \\
\text { R:CCATTACATAAGGTCTTGGGTTCAA }\end{array}$ & $\mathrm{CAA}_{(6)}$ & $128-134$ & 59 & 13 & 0.5667 & 0.8932 & 0.375 & 0.6103 & KX530281 \\
\hline DZC356 & $\begin{array}{l}\text { F:GAGGTCACTGTGAACATCACGTTT } \\
\text { R:GGATTTCATTCGTGAAAAGTTAGGG }\end{array}$ & $\mathrm{CA}_{(15)}$ & $140-160$ & 59 & 13 & 0.7333 & 0.9169 & 0.530 & 0.0773 & KX530282 \\
\hline DZC429 & $\begin{array}{l}\text { F:GTGATTGCGACCGAGTCATATTG } \\
\text { R:AGTCTGCTCGGAAGGAAAAAAGAAA }\end{array}$ & $\mathrm{GT}_{(11)}$ & $140-146$ & 59 & 8 & 0.5000 & 0.8299 & 0.611 & 0.0259 & KX530283 \\
\hline$\overline{\mathrm{DZC} 408}$ & $\begin{array}{l}\text { F:ACTTTTGTATGTCATGGTCTGCGA } \\
\text { R:TGTGACTGACAGCAGAGGTACTCA }\end{array}$ & $\mathrm{TAG}_{(6)}$ & $138-150$ & 59 & 14 & 0.9000 & 0.8847 & 0.449 & 0.0043 & KX530284 \\
\hline$\overline{\mathrm{DZC110}}$ & $\begin{array}{l}\text { F:TATAAGGGCATGGGGCATAGTTTA } \\
\text { R:AGATAATGAAATCATAATAGCAAATGCG }\end{array}$ & $\mathrm{AG}_{(12)}$ & $146-166$ & 57 & 11 & 0.7333 & 0.8678 & 0.489 & 0.0016 & KX530285 \\
\hline DZC152 & \begin{tabular}{|l} 
F:TTATGGTGCTTTTTGTGGTGTTTG \\
R:AAAACCATGCAGTGAGATGACCTT
\end{tabular} & $\operatorname{TAA}_{(5)}$ & 150-156 & 54 & 11 & 0.9333 & 0.8582 & 0.255 & 0.6735 & KX530286 \\
\hline
\end{tabular}

Continued on next page

Genetics and Molecular Research 16 (1): gmr16019621 


\begin{tabular}{|c|c|c|c|c|c|c|c|c|c|c|}
\hline Locus & Primer sequence (5'-3') & Repeat motif & Fragment size (bp) & $\mathrm{Ta}\left({ }^{\circ} \mathrm{C}\right)$ & $N_{\mathrm{A}}$ & $H_{0}$ & $H_{\mathrm{E}}$ & PIC & $\begin{array}{c}\text { v value } \\
\end{array}$ & GenBank accession No. \\
\hline DZC166 & $\begin{array}{l}\text { F:TTCAGCAACATCACAAGCAATACA } \\
\text { R:CATCATCACCATTATCTGTGCCAT }\end{array}$ & $\mathrm{ATG}_{(10)}$ & $150-168$ & 54 & 11 & 0.4333 & 0.7678 & 0.694 & 0.1329 & KX530287 \\
\hline DZC108 & $\begin{array}{l}\text { F:GAGCCACAAGGTCTTCCTTACAAA } \\
\text { R:ACATTTTCAAAGCTAGGAAGTGGG }\end{array}$ & $\operatorname{TTG}_{(7)}$ & $140-148$ & 60 & 15 & 0.8667 & 0.9119 & 0.729 & 0.0010 & KX530288 \\
\hline DZC192 & $\begin{array}{l}\text { F:AGACGATTTTCAGAAGTCAAACGC } \\
\text { R:CAGGCTTGACGTCACATAACTTTG }\end{array}$ & $\mathrm{AT}_{(10)}$ & $126-138$ & 60 & 23 & 0.8667 & 0.9480 & 0.671 & 0.0362 & KX530289 \\
\hline DZC119 & $\begin{array}{l}\text { F:TGGCATGGAGAGTTTTGAGTAATA } \\
\text { R:CAGAAGCGCTTATCAATCTTCTTT }\end{array}$ & $\mathrm{TTG}_{(23)}$ & $120-130$ & 55 & 17 & 0.7667 & 0.9220 & 0.679 & 0.0190 & KX530290 \\
\hline$\overline{\mathrm{DZC} 120}$ & $\begin{array}{l}\text { F:GTTTGAAAAGAATTACAGGCAGCA } \\
\text { R:ATGCATTACTTCATCGTTGAGCAG }\end{array}$ & $\mathrm{AC}_{(13)}$ & $152-158$ & 55 & 7 & 0.1667 & 0.4051 & 0.362 & 0.0054 & KX530291 \\
\hline DZC117 & $\begin{array}{l}\text { F:CGATGAGTATGTTCACGATTGCAT } \\
\text { R:TGCATGATGATAAATAATGGGGAC }\end{array}$ & $\mathrm{GA}_{(12)}$ & $120-138$ & 55 & 5 & 0.1667 & 0.2763 & 0.752 & 0.0140 & KX530292 \\
\hline DZC204 & $\begin{array}{l}\text { F:CTGTCCCACCAATAACCAAACTCT } \\
\text { R:ACATTTTACCAAGCTGGAAACGAAA }\end{array}$ & $\mathrm{TCTG}_{(4)}$ & $118-128$ & 54 & 12 & 0.6667 & 0.8847 & 0.381 & 0.0058 & KX530293 \\
\hline DZC227 & \begin{tabular}{|l|} 
F:ATGCTAAACAAGTCGAACCACCTT \\
R:AAATATTGCCGAAGCGACTGTAAA
\end{tabular} & $\mathrm{TGACT}_{(5)}$ & $100-114$ & 54 & 2 & 0.1000 & 0.0966 & 0.258 & 0.0798 & KX530294 \\
\hline DZC250 & $\begin{array}{l}\text { F:CTCATGCCACCATTAGTCATATCG } \\
\text { R:ACATGAAGGAAGGAAGAGTACGGA }\end{array}$ & $\mathrm{TC}_{(13)}$ & $130-144$ & 60 & 13 & 0.9667 & 0.9119 & 0.538 & 0.0107 & KX530295 \\
\hline DZC220 & \begin{tabular}{|l} 
F:GGCAAAATACCGTTTAATGCGTAA \\
R:TTAACAGGGGTAATTTGTTTGCG
\end{tabular} & TGTAT $_{(5)}$ & $112-122$ & 54 & 11 & 0.4667 & 0.8497 & 0.606 & 0.0746 & KX530296 \\
\hline DZC211 & $\begin{array}{l}\text { F:TGCAAACATGTAAGCTGTCAAACTT } \\
\text { R:TGCATGATATTTAATGGAAATTGGAA }\end{array}$ & $\mathrm{AAC}_{(6)}$ & $142-154$ & 54 & 8 & 0.6667 & 0.8446 & 0.495 & 0.0418 & KX530297 \\
\hline DZC255 & $\begin{array}{l}\text { F:AAATATTCAGACGATGGAAAAAGGAAA } \\
\text { R:TTAACGCCGAAGGTATGTGAACTT }\end{array}$ & $\mathrm{AG}_{(11)}$ & $100-108$ & 60 & 7 & 1.0000 & 0.7051 & 0.617 & 0.0721 & KX530298 \\
\hline DZC379 & \begin{tabular}{|l|} 
F:CGATCCTTTTGTCGACTGACTCTT \\
R:TAAGGAAGGACAGAATCCCACATC
\end{tabular} & $\operatorname{TTC}_{(8)}$ & $144-158$ & 60 & 11 & 0.9333 & 0.8412 & 0.618 & 0.0005 & KX530299 \\
\hline DZC371 & \begin{tabular}{|l|} 
F:AAACCCACCTTGGTCGATTTTAG \\
R:GAGAAAGTGAGCCAATGTATTGATAGC
\end{tabular} & $\mathrm{TGT}_{(5)}$ & $148-156$ & 64 & 2 & 0.3333 & 0.4520 & 0.431 & 0.0191 & KX530300 \\
\hline DZC302 & $\begin{array}{l}\text { F:AATCTGGATCAGACATCAGTCAAAA } \\
\text { R:TGAATGTCAAACGCATAGTTTTAGC }\end{array}$ & $\mathrm{GT}_{(13)}$ & $236-250$ & 55 & 5 & 0.5000 & 0.5655 & 0.427 & 0.0094 & KX530301 \\
\hline DZC309 & $\begin{array}{l}\text { F:AGGGATATGGTAATGATGGTTGGA } \\
\text { R:GCAGCGGCAGCATAATGTATC }\end{array}$ & $\mathrm{TGA}_{(14)}$ & $138-150$ & 61 & 7 & 0.3667 & 0.3842 & 0.675 & $0.0000^{*}$ & KX530302 \\
\hline DZC348 & $\begin{array}{l}\text { F:ACGTAGCCGCTTAAAAGTTATGCC } \\
\text { R:TTCGGATCCGTAGCCTATTACGTT }\end{array}$ & $\mathrm{TG}_{(11)}$ & $200-214$ & 64 & 5 & 0.2667 & 0.3011 & 0.499 & $0.0000^{*}$ & KX530303 \\
\hline DZC274 & $\begin{array}{l}\text { F:TGACGGAATAAGTGTGCTTGATGT } \\
\text { R:AACCAATGAATCAACAACTCCCAG }\end{array}$ & $\mathrm{GAT}_{(8)}$ & $148-156$ & 55 & 7 & 0.6333 & 0.5910 & 0.399 & 0.3246 & KX530304 \\
\hline DZC355 & $\begin{array}{l}\text { F:CCTCCTCGCCTCATTATCATTATC } \\
\text { R:CAAGTATTGGATGATCCCCTCTGT }\end{array}$ & $\mathrm{TCA}_{(23)}$ & $140-154$ & 60 & 7 & 0.5667 & 0.7701 & 0.305 & 0.0204 & KX530305 \\
\hline DZC276 & \begin{tabular}{|l|} 
F:TTTCTGTGTCACCTGTTTAAAGGCA \\
R:TTTGGAACATATGTGTCACCTGCT
\end{tabular} & $\operatorname{TCTG}_{(8)}$ & $106-112$ & 60 & 2 & 0.5000 & 0.5079 & 0.421 & 0.2671 & KX530306 \\
\hline DZC431 & $\begin{array}{l}\text { F:AACAATCAATCATGCAGAAAGCAA } \\
\text { R:TATTAGAATAGCGCCACCTCCAAC }\end{array}$ & $\mathrm{CG}_{(7)}$ & $152-158$ & 55 & 4 & 0.7667 & 0.5938 & 0.375 & 0.2036 & KX530307 \\
\hline DZC167 & $\begin{array}{l}\text { F:TGTTACGCATATGTTTTGTTTCGC } \\
\text { R:GAAATATGATGATGAGCACTTTGGG }\end{array}$ & $\operatorname{TATG}_{(12)}$ & $138-150$ & 54 & 4 & 0.4333 & 0.6785 & 0.748 & $0.0000^{*}$ & KX530308 \\
\hline DZC260 & $\begin{array}{l}\text { F:GTGATGAGGATTTAATGGGTGGAG } \\
\text { R:CAACATTTGATTCTGGTTCAGTGC }\end{array}$ & $\mathrm{CCT}_{(4)}$ & 100-110 & 59 & 4 & 0.3333 & 0.3915 & 0.321 & 0.0374 & KX530309 \\
\hline$\overline{\mathrm{DZC}} 360$ & $\begin{array}{l}\text { F:TACGGTCCGCCATTAATTGATTT } \\
\text { R:GAACGCAAAGATAACCATCAGAGC }\end{array}$ & $\mathrm{TGT}_{(10)}$ & $250-268$ & 57 & 9 & 0.7333 & 0.7944 & 0.765 & $0.0000^{*}$ & KX530310 \\
\hline$\overline{\text { DZC420 }}$ & $\begin{array}{l}\text { F:CCACTGTAACCCAGTTTTTCAACA } \\
\text { R:CATCTCCTGAATGGATTTTAACCA }\end{array}$ & $\operatorname{TGTT}_{(5)}$ & $130-146$ & 57 & 6 & 0.4000 & 0.6712 & 0.235 & 0.1020 & KX530311 \\
\hline$\overline{\text { DZC444 }}$ & $\begin{array}{l}\text { F:GGCGAAGTATACATATACACGACAAGG } \\
\text { R:AGGCACTCAAAATGGAAATCAAAC }\end{array}$ & $\mathrm{GT}_{(14)}$ & $196-216$ & 57 & 5 & 0.3333 & 0.5350 & 0.707 & 0.0224 & KX530312 \\
\hline $\begin{array}{l}\mathrm{DZC} 128 \\
\end{array}$ & $\begin{array}{l}\text { F:AACAACAATTTCAACTGCTTCACAAA } \\
\text { R:CACGGTTTCACGGATATCTTCTACA }\end{array}$ & $\mathrm{TAT}_{(6)}$ & 180-194 & 59 & 5 & 0.2000 & 0.3266 & 0.479 & 0.0579 & KX530313 \\
\hline$\overline{\mathrm{DZC} 132}$ & $\begin{array}{l}\text { F:CAGCGTCTGGAAATACGTTAATATG } \\
\text { R:TGAATGTAGACTATGCCTGGTATTGA }\end{array}$ & $\mathrm{TGGA}_{(6)}$ & $146-168$ & 58 & 4 & 0.3000 & 0.3226 & 0.267 & 0.1154 & KX530314 \\
\hline$\overline{\mathrm{DZC} 312}$ & $\begin{array}{l}\text { F:AATCTTAAAATTTGCCGCCTTCTG } \\
\text { R:TCAAATATTCAAGACGATATCCATGAAA }\end{array}$ & $\mathrm{TG}_{(18)}$ & $158-170$ & 58 & 9 & 0.3667 & 0.8028 & 0.535 & $0.0000^{*}$ & KX530315 \\
\hline $\begin{array}{l}\mathrm{DZC} 107 \\
\end{array}$ & $\begin{array}{l}\text { F:CTGATCCGTCGAAAGGGTAAAGTA } \\
\text { R:TGTTAAAAGATTATCCATTTCATCATGG }\end{array}$ & $\mathrm{GAT}_{(8)}$ & $150-162$ & 58 & 5 & 0.2000 & 0.2475 & 0.398 & 0.0026 & KX530316 \\
\hline DZC396 & $\begin{array}{l}\text { F:CGGTGTACTTGTGGATTGCATAGA } \\
\text { R:TGAAAGAGATGAACGCAAAAATGA }\end{array}$ & $\operatorname{ATTC}_{(5)}$ & $150-168$ & 55 & 7 & 0.5333 & 0.7588 & 0.423 & 0.0011 & KX530317 \\
\hline$\overline{\mathrm{DZC}} 380$ & \begin{tabular}{|l|} 
F:TGTCCGAGCTATTAACATTATTTCCA \\
R:CTTTTCTGCGAAGTTGTTGGTTTT
\end{tabular} & $\mathrm{TGT}_{(9)}$ & $130-148$ & 57 & 6 & 0.4000 & 0.5141 & 0.460 & 0.2446 & KX530318 \\
\hline $\begin{array}{l}\text { DZC244 } \\
\end{array}$ & \begin{tabular}{|l|} 
F:GGTACCGTCAAGGATGATATCGAG \\
R:TTCAAAATGGGTCAATCAATGCTA
\end{tabular} & $\mathrm{TC}_{(12)}$ & 116-126 & 55 & 5 & 0.1333 & 0.5898 & 0.417 & 0.2550 & KX530319 \\
\hline$\overline{\mathrm{DZC} 424}$ & $\begin{array}{l}\text { F:AACAGGTGGCCTATATCCTTCGTA } \\
\text { R:TTCATGCCTTTTATACAGCGTTTG }\end{array}$ & $\mathrm{TA}_{(8)}$ & 116-124 & 63 & 3 & 0.5000 & 0.5237 & 0.456 & 0.0162 & KX530320 \\
\hline DZC127 & $\begin{array}{l}\text { F:ACTCTTTGCATTCAAGCTCCACAC } \\
\text { R:CATTGGAGTCGGTGCTGATAATTT }\end{array}$ & $\mathrm{GT}_{(12)}$ & $210-230$ & 60 & 5 & 0.4333 & 0.4480 & 0.559 & $0.0000^{*}$ & KX530321 \\
\hline$\overline{\text { DZC419 }}$ & $\begin{array}{l}\text { F:GCGGATTGAATCTCATTACACACA } \\
\text { R:CGAACAGAAAAATCGTTTCTAGGC }\end{array}$ & $\mathrm{CT}_{(10)}$ & $120-136$ & 63 & 8 & 0.3667 & 0.5955 & 0.657 & $0.0000^{*}$ & KX530322 \\
\hline
\end{tabular}

$\mathrm{Ta}=$ annealing temperature; $N_{\mathrm{A}}=$ number of alleles per locus; $H_{\mathrm{O}}=$ observed heterozygosity; $H_{\mathrm{E}}=$ expected heterozygosity; PIC = polymorphism information content; *significant deviations from Hardy-Weinberg equilibrium $(\mathrm{P}<0.05 / 105)$.

Compared to the traditional enrichment techniques and Sanger sequencing method, the development of 105 loci using the Illumina pair-end shotgun sequencing approach was a success. With 105 polymorphic microsatellite loci now developed and characterized in this

Genetics and Molecular Research 16 (1): gmr16019621 
paper, fine-scale genetic diversity and structure studies, as well as selection and mapping studies will be possible for $S$. grandis, which contribute to make reasonable recovery and management plans guiding aquaculture programs.

\section{Conflicts of interest}

The authors declare no conflict of interest.

\section{ACKNOWLEDGMENTS}

Research supported by grants from the Shandong Provincial Agriculture Thoroughbred Project to Xiangquan Liu and the Innovation Project of Guangxi Graduate Education (\#YCBZ2015007).

\section{REFERENCES}

Botstein D, White RL, Skolnick M and Davis RW (1980). Construction of a genetic linkage map in man using restriction fragment length polymorphisms. Am. J. Hum. Genet. 32: 314-331.

Dai CJ (2002). Evaluation on nutrition components of the soft part in Solen grandis. J. Jimei Univ. 7: 304-308.

Dieringer D and Schlötterer C (2003). Microsatellite analyser (MSA): a platform independent analysis tool for large microsatellite data sets. Mol. Ecol. Notes 3: 167-169. http://dx.doi.org/10.1046/j.1471-8286.2003.00351.x

Ji P, Liu G, Xu J, Wang X, et al. (2012). Characterization of common carp transcriptome: sequencing, de novo assembly, annotation and comparative genomics. PLoS One 7: e35152. http://dx.doi.org/10.1371/journal.pone.0035152

Jiang HL, Feng YW, Wu XP, Liu XQ, et al. (2016). Microsatellite records for volume 8, issue 1. Conserv. Genet. Resour. 8: 43-81. http://dx.doi.org/10.1007/s12686-016-0522-2

Jiao Y, Jia HM, Li XW, Chai ML, et al. (2012). Development of simple sequence repeat (SSR) markers from a genome survey of Chinese bayberry (Myrica rubra). BMC Genomics 13: 201. http://dx.doi.org/10.1186/1471-2164-13-201

Li Q and Wan JM (2005). SSRHunter: development of a local searching software for SSR sites. Yi Chuan 27: 808-810.

Li Q, Yu H and Yu RH (2006). Genetic variability assessed by microsatellite in cultured population of Pacific oyster (Crassostera gigas) in China. Aquaculture 259: 95-102. http://dx.doi.org/10.1016/j.aquaculture.2006.05.030

Marshall TC, Slate J, Kruuk LEB and Pemberton JM (1998). Statistical confidence for likelihood-based paternity inference in natural populations. Mol. Ecol. 7: 639-655. http://dx.doi.org/10.1046/j.1365-294x.1998.00374.x

Powell W, Morgante M, Andre C, Hanafey M, et al. (1996). The comparison of RFLP, RAPD, AFLP and SSR (microsatellite) markers for germplasm analysis. Mol. Breed. 2: 225-238. http://dx.doi.org/10.1007/BF00564200

Rousset F (2008). genepop'007: a complete re-implementation of the genepop software for Windows and Linux. Mol. Ecol. Resour. 8: 103-106. http://dx.doi.org/10.1111/j.1471-8286.2007.01931.x

Yang DJ and Wang YL (1996). Marine Invertebrate in Northern China. High Education Press, Beijing.

Yuan Y, Li Q, Kong LF and Zheng XD (2010). Isolation and characterization of polymorphic microsatellite loci in the grand jackknife clam Solen grandis (Bivalvia: Veneroida). Genes Genomics 32: 191-197. http://dx.doi.org/10.1007/ $\underline{\text { s13258-009-0780-4 }}$

Genetics and Molecular Research 16 (1): gmr16019621 